\title{
T4a Stage Finding
}

National Cancer Institute

\section{Source}

National Cancer Institute. T4a Stage Finding. NCI Thesaurus. Code C48733.

A general term that refers to a TNM finding of a primary tumor with direct invasion of adjacent structures. The definition of T4a TNM finding depends on the specific type of cancer that it refers to; for example, for bladder cancer it refers to a primary tumor that invades the prostatic stroma, uterus, and vagina; for gastric cancer it refers to a primary tumor that invades the serosa (visceral peritoneum); for colorectal cancer it refers to a primary tumor that penetrates to the surface of the visceral peritoneum. 